Open Access

\title{
CoDiab-VD: protocol of a prospective population-based cohort study on diabetes care in Switzerland
}

Emilie Zuercher, Julie Bordet, Bernard Burnand and Isabelle Peytremann-Bridevaux*

\begin{abstract}
Background: Diabetes represents an increasing health burden worldwide. In 2010, the Public Health Department of the canton of Vaud (Switzerland) launched a regional diabetes programme entitled "Programme cantonal Diabète" $(P C D)$, with the objectives to both decrease the incidence of diabetes and improve care for patients with diabetes. The cohort entitled CoDiab-VD emerged from that programme. It specifically aimed at following quality of diabetes care over time, at evaluating the coverage of the PCD within this canton and at assessing the impact of the PCD on care of patients with diabetes.
\end{abstract}

Methods/Design: The cohort CoDiab-VD is a prospective population-based cohort study. Patients with diabetes were recruited in two waves (autumn 2011 - summer 2012) through community pharmacies. Eligible participants were non-institutionalised adult patients ( $\geq 18$ years) with diabetes diagnosed for at least one year, residing in the canton of Vaud and coming to a participating pharmacy with a diabetes-related prescription. Women with gestational diabetes, people with obvious cognitive impairment or insufficient command of French were not eligible. Self-reported data collected, included the following primary outcomes: processes-of-care indicators (annual checks) and outcomes of care such as $\mathrm{HbA1C}$, (health-related) quality of life measures (Short Form-12 Health Survey SF-12, Audit of Diabetes-Dependent Quality of Life 19 - ADDQoL) and Patient Assessment of Chronic Illness Care (PACIC). Data on diabetes, health status, healthcare utilisation, health behaviour, self-management activities and support, knowledge of, or participation to, campaigns/activities proposed by the PCD, and socio-demographics were also obtained. For consenting participants, physicians provided few additional pieces of information about processes and laboratory results.

Participants will be followed once a year, via a mailed self-report questionnaire. The core of the follow-up questionnaires will be similar to the baseline one, with the addition of thematic modules adapting to the development of the PCD. Physicians will be contacted every 2 years.

Discussion: CoDiab-VD will allow obtaining a broad picture of the care of patients with diabetes, as well as their needs regarding their chronic condition. The data will be used to evaluate the PCD and help prioritise targeted actions.

Trial registration: This study is registered with ClinicalTrials.gov, identifier NCT01902043, July 9, 2013.

\footnotetext{
* Correspondence: Isabelle.Peytremann-Bridevaux@chuv.ch

Institute of social and preventive medicine (IUMSP), Lausanne University

Hospital, Lausanne, Switzerland
}

(c) 2015 Zuercher et al. Open Access This article is distributed under the terms of the Creative Commons Attribution 4.0 International License (http://creativecommons.org/licenses/by/4.0/), which permits unrestricted use, distribution, and reproduction in any medium, provided you give appropriate credit to the original author(s) and the source, provide a link to the Creative Commons license, and indicate if changes were made. The Creative Commons Public Domain Dedication waiver (http://creativecommons.org/publicdomain/zero/1.0/) applies to the data made available in this article, unless otherwise stated. 


\section{Background}

Worldwide, chronic diseases constitute a major burden for communities in terms of morbidity, disability and mortality. Their care requires collaboration between healthcare providers, teamwork, training in self-management and the use of evidence based-medicine. However, such care processes are complex and often suboptimal. Thus, chronic disease management (CDM) strategies have been developed as a means of reorganizing healthcare systems and medical treatment for chronic diseases [1-4].

Diabetes is one of the most common chronic diseases, with an estimated number of affected persons of more than 550 million for 2030 [5]. In the canton of Vaud, a Swiss state of $\sim 720.000$ residents (approximately $10 \%$ of the Swiss population), a recent population-based study has shown a prevalence of diabetes of about $7 \%$ [6]. In 2010, the Public Health Department of the canton of Vaud initiated the development of a regional programme entitled "Programme cantonal Diabète" (PcD), with both the aim to decrease the prevalence of diabetes in that canton and improve care for patients with diabetes [7]. The political will and support for this innovative programme, at the level of a whole canton, is unique in Switzerland.

Evaluation of CDM programmes is essential to assess their implementation and determine the benefits and effectiveness of their development [8, 9]. Randomized controlled trials (RCT) may not be the most suitable design for evaluating complex interventions such as the $\mathrm{PcD}$, since such interventions are very much contextdependent [10]. In addition, there might be practical difficulties to include control sites, and the resources and costs incurred may be substantial. Moreover, when implemented at a macro level, with no direct inclusion of patients, RCT cannot be considered as an evaluation alternative. Scientifically sound evaluation methods that remain also practical in routine settings are needed; cohort studies may represent such an opportunity.

To assess the impact of the implementation of the PcD on health and care outcomes as well as to obtain a comprehensive picture of patients with diabetes residing in the canton of Vaud, and their care, we opted for a prospective cohort study design. After the baseline data collection, participants will be followed-up annually to assess the evolution of the quality of care over time, the coverage of the PcD within that canton and the impact of the $\mathrm{PcD}$ on patients' care.

\section{Methods/Design}

\section{Design}

The CoDiab-VD is a prospective population-based cohort study launched in 2011 in the canton of Vaud/ Switzerland, conducted by the Institute of Social and Preventive Medicine (IUMSP) of the Lausanne University Hospital. Baseline data were collected during a two- wave recruitment period in the autumn of 2011 and in the summer of 2012 (Fig. 1). Patients' follow-ups will be set up yearly (Fig. 2).

\section{Study population \\ Sampling frame}

Randomly selected community-based pharmacies registered in the canton of Vaud were contacted and asked to participate in the recruitment of patients with diabetes [11]. During a 6 week period, participating pharmacies had to propose the survey to 40 consecutive persons visiting the pharmacy with a prescription for diabetes-related treatment and/or equipment (oral anti-diabetic medications, insulin, glycemic strips or glucose meter). Pharmacists checked patients' eligibility and briefly described the context and aims of the study. The baseline questionnaire package (information letter, questionnaire, prepaid reply envelope) was given to eligible patients accepting to take it, to complete it at home, and to return it to the investigators by regular mail. Pharmacists did not record contact details of eligible participants because of time and organisation constraints; therefore potentially participating patients with diabetes could not be reminded to send back the questionnaire. For the same reason, the characteristics of nonparticipants could not be recorded.

\section{Inclusion criteria}

Patients were eligible if they came to a participating pharmacy with a diabetes-related prescription, and were noninstitutionalised adults ( $\geq 18$ years) reporting a diagnosis of diabetes for at least 1 year, residing in the canton of Vaud and able to provide written informed consent.

\section{Exclusion criteria}

Exclusion criteria at baseline were gestational diabetes, obvious cognitive impairment, or insufficient level of French to understand and complete the self-report questionnaire.

\section{Sample size}

The number of patients with diabetes to include in the study was estimated to obtain good precision (i.e. confidence interval width) around the following primary outcomes at baseline: mean $\mathrm{HbA1C}$ value, mean Physical and Mental component scores (PCS and MCS) of the Short Form-12 Health Survey (SF-12), mean Patient Assessment of Chronic Illness Care (PACIC) global score, and percentage of patients receiving recommended annual processesof-care. Considering the clustering of data by pharmacies (40 pharmacies, each recruiting 15 patients, intra-class correlation 0.05 , alpha 0.05 , beta 0.2 ), the recruitment of 600 participants was deemed appropriate. This sample size was also large enough to detect a $0.5 \%$ point decrease in $\mathrm{HbA1C}$ between two time points. In addition, it was 

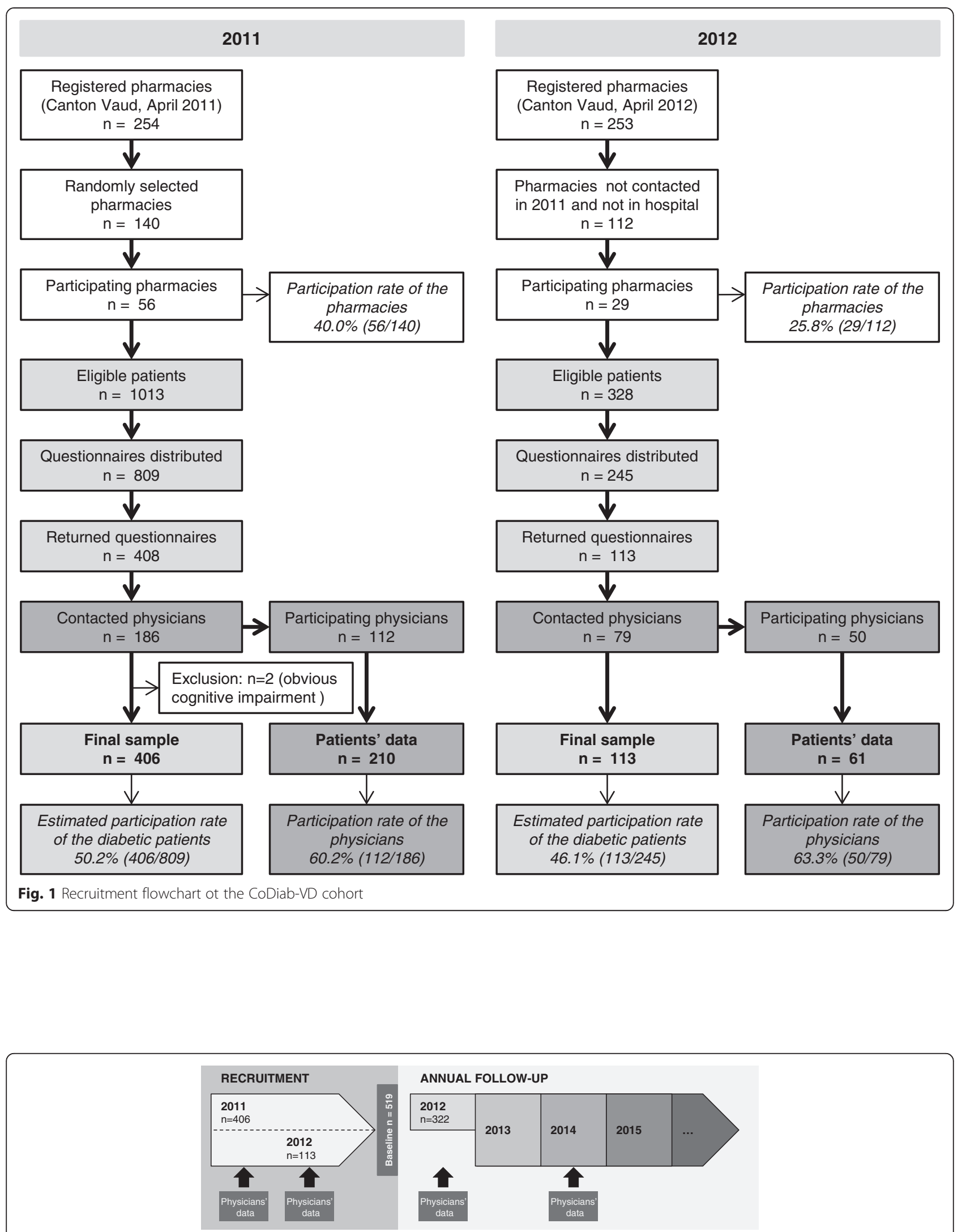

Fig. 2 Study design of the CoDiab-VD cohort 
above the sample size required for such a change considering repeated measures within a same individual.

\section{Baseline measures}

\section{Patients' questionnaire}

The baseline questionnaire was designed to encompass several aspects of disease, care and life of people living with diabetes. It targeted the following areas: diabetes status, diabetes management and quality of care, health-related quality of life (HRQoL) and quality of life (QoL), health services utilisation, health status and health habits, self-management activities and support, socio-demographics. An overview of all variables collected is shown in Table 1, and a comprehensive description of corresponding questionnaires and answer categories is provided in the Additional file 1.

\section{Selection of indicators}

The selection of quality indicators followed a three-step procedure. First, we searched the published and grey literature for frequently used diabetes quality of care indicators and population-based surveys exploring the quality of care of patients with diabetes. Then, we identified published clinical practice guidelines on diabetes care and considered those most recently updated and used by a group of regional partners adapting diabetes guidelines for Switzerland. The guidelines developed by the following agencies were considered: National Institute for Health and Clinical Excellence (NICE), Scottish Intercollegiate Guidelines Network (SIGN), International Diabetes Foundation (IDF), Canadian Diabetes Association (CDA), American Diabetes Association (ADA), Haute Autorité de Surveillance (HAS). Finally, we took into account three criteria: clinical significance, practical relevance (feasibility), and reliability/validity of the measurement. When several instruments existed, we considered their validity, their length, and the existence of a French version. We favoured instruments which presented the best balance of those latter criteria.

\section{Primary outcomes: processes and outcomes quality of care indicators}

We considered as primary outcomes all usually recommended diabetes processes-of-care indicators, as well as the following outcomes of care indicators: HbA1C levels, as an intermediary outcome associated with future development of diabetes complications [12-14], HRQoL and QoL as measured using a generic tool (SF-12) [15] and a diabetes-specific quality of life tool (Audit of Diabetes-Dependent Quality of Life 19 - ADDQoL) [16], respectively, and patient assessment of chronic care, i.e. how care is congruent with the Chronic Care Model (PACIC) $[17,18]$.

\section{Exposure variables}

In the 2012 baseline questionnaire, we also added a small number of questions regarding knowledge and participation in the few activities proposed that year by the PcD. These questions were only asked to patients recruited in 2012 in order to describe possible differences in terms of exposition to, and awareness of, the PcD, because patients were recruited over two different time periods.

\section{Other variables of interest}

The other variables of interest considered in the baseline questionnaire are briefly described thereafter; more details are provided in Table 1 and Additional file 1.

- Diabetes characteristics and related complications;

- Medication adherence using the Morisky medication adherence questionnaire [19];

- Healthcare utilisation within the past 12 months: ambulatory care visits, emergency visits, hospitalisation, home care services, domestic home support;

- Care foregone because of costs;

- Health status and health habits: anthropometric measures (weight and height allowing the calculation of the body mass index (BMI)), smoking status, alcohol consumption using the AUDIT-C questionnaire [20], levels of physical activity using questions from the Swiss Health Survey [21], depression screening using two validated questions [22] and comorbidities;

- Self-management activities and support measures: home glucose self-monitoring, HbA1C knowledge, participation in diabetes education classes, membership of the local diabetes association (Association Vaudoise du Diabète - AVD), knowledge of the "Diabetes Passport" (a small booklet with data, information and reminders) and if it was known, whether it was used;

- "Self-efficacy" measure, which was developed de novo because available instruments were either not appropriate from our point of view, or too long or did not have a French version. We were interested in exploring how easy/difficult it was for patients to manage their diabetes, overall, and also specifically, regarding the daily management of physical activity, medication and diet;

- Level and source of information about diabetes;

- Support and satisfaction from the healthcare team or from the members of the social network, if any;

- Overall satisfaction with current care and care recommendation to others;

- Patients' socio-demographic characteristics: age, gender, nationality, place of residence, marital status, family size, education, employment, household income and insurance status. 
Table 1 Overview of the variables, measures and instruments used in the patient self-completed questionnaire

Section
Primary outcomes: processes and
outcomes quality of care
indicators
Diabetes management

Receipt of recommended processes-of-care

$\mathrm{HbA} 1 \mathrm{C}$

Health-related quality of life $(\mathrm{HRQOL})$ and Quality of life (QoL)

Generic HRQoL

Diabetes-specific QoL

Congruency of care with the

Patient assessment of chronic care

Chronic Care Model (CCM)

Exposure variables

Programme cantonal Diabète ${ }^{a}$

Knowledge of/participation in PcD activities/projects

Other variables of interest

Diabetes

Diabetes-related complications

Diabetes management

Health services utilisation

Health status and health habits

Self-management activities and support

Ambulatory care visits, emergency visits, hospitalisation

Home care services, domestic home support

Foregoing care because of costs

Anthropometric values

Smoking

Alcohol consumption

Physical activity levels

Depression screening

Comorbidities

Home glucose self-monitoring
Instruments, details

1) Past 12 months: HbA1C check, blood pressure measurement, weight measurement, lipid profile, diabetic foot examination, urine test for microalbuminuria, eye examination by ophtalmologist, influenza vaccination 2) Anytime: physical activity recommendations, diet recommendations Last $\mathrm{HbA} 1 \mathrm{C}$ value

SF-12 [15]

ADDQoL [16]

PACIC $[17,18]$
Characteristics of the disease

Type of diabetes, disease duration, treatment (drugs)

List of following complications: ischemic heart diseases, stroke, retinopathy, chronic kidney disease (CKD) without dialysis, CKD with dialysis or kidney transplant, neuropathy, foot ulcer, lower limb amputation, severe hypoor hyperglycemia

Morisky Medication Adherence Questionnaire [19]

Utilisation during past 12 months

HbA1C knowledge

Participation in diabetes education courses

Membership in the local diabetes association

Knowledge and use of the "Diabetes Passport"

"Self-efficacy"

Level and source of information about diabetes
Received help during past 12 months

Foregoing care during past 12 months

Weight, height

Smoking status, duration of smoking, smoking products, average number of cigarettes smoked per day, medical advice on smoking cessation

Questions from the Swiss Health Survey [21]

Two validated questions for the screening of depression [22]

List of following chronic diseases: heart disease (heart failure, valve disease, heart muscle disease), chronic lung disease (asthma, chronic bronchitis, emphysema), osteoporosis, osteoarthritis or arthritis; cancer or malignancy or lymphoma (with the exception of skin cancer), gastric or duodenal ulcer, depression, Parkinson disease, hypertension, hyperlipidemia, other chronic condition

Level of easiness/difficulty to manage diabetes generally, and regarding physical activity, diet, and medication
AUDIT-C questionnaire [20] 
Table 1 Overview of the variables, measures and instruments used in the patient self-completed questionnaire (Continued)

\begin{tabular}{|c|c|c|}
\hline & $\begin{array}{l}\text { Support and satisfaction from } \\
\text { healthcare team and social network }\end{array}$ & \\
\hline & $\begin{array}{l}\text { Diabetes care satisfaction and } \\
\text { recommandation of their care to } \\
\text { others }\end{array}$ & \\
\hline \multirow[t]{2}{*}{ Socio-demographics } & Characteristics of the participants & Age, gender \\
\hline & Socio-economic status & $\begin{array}{l}\text { Marital status, family size, household income, education, employment, } \\
\text { insurance status, place of residence, nationality }\end{array}$ \\
\hline
\end{tabular}

HRQoL health-related quality of life, SF-12 short form-12 Health Survey, ADDQoL audit of diabetes-dependent quality of life 19, CCM chronic care model, PACIC patient assessment of chronic illness care

${ }^{\mathrm{a}}$ Since the 2012 recruitment

${ }^{\mathrm{b}} \mathrm{A}$ small booklet with data, information and reminders

\section{Pretest of the questionnaire}

Before the recruitment, we pretested the questionnaire among 12 patients with diabetes to ensure the understanding and acceptability of the instructions and of the questions, as well as to measure the completion time of the questionnaire as a whole.

\section{Treating physicians' questionnaire}

If participants agreed to share their physicians' contact details, the physicians were contacted and asked to complete a questionnaire on their patients' clinical, laboratory and processes-of-care values, as well as a personal questionnaire including some of their characteristics and characteristics of their practice (Table 2).

\section{Patients' information}

Physicians were asked to report the type of diabetes (type 1, type 2, other:specify), the last value and date of the following measures: $\mathrm{HbA1C}$, lipid profile, serum creatinine (since the 2012 follow-up), urine microalbuminurie, blood pressure, weight and height. They were also asked to report whether the following processes-of-care were done or not as well as the date of the examination: foot examination, eye examination by ophthalmologist, influenza vaccination. Finally, they had to assess satisfaction with their patients' care and management (5-point scale: entirely satisfied to not at all satisfied), as well as describe the barriers they were facing which prevented them to better take care of their patients (no barrier, insufficient time during consultation, other health problems of the patient, lack of motivation of the patient, health insurance reimbursement or insurance problem, language or cultural barrier, other).

\section{Physicians' characteristics}

Physicians' characteristics encompassed age, gender of the physician, year of medical diploma, year of start in private practice, and board certification type. We also asked a few questions allowing the characterization of their practice: location and type, activity rate, participation in quality programmes/circles.

\section{Follow-up}

\section{Follow-up process}

Participants will be followed annually by mail questionnaires (information letter, paper questionnaire and prepaid reply envelope). To maximise retention of participants in the cohort, we will send a postcard reminder 2 weeks after the first mailing to non-respondents and a complete reminder package again 2 weeks later (questionnaire, information letter and prepaid reply envelope). Persistent nonrespondents will be contacted by telephone; a minimum of

Table 2 Overview of the variables, measures and instruments used in the Physician-completed questionnaires

\begin{tabular}{|c|c|c|}
\hline Section & Variables & Details/Answers \\
\hline \multicolumn{3}{|l|}{ Patients' information } \\
\hline Diabetes & Diabetes type & \\
\hline Laboratory results & $\begin{array}{l}\text { HbA1C, lipid profile, serum creatinine }{ }^{a} \text {, urine microalbuminuria, blood pressure, weight, } \\
\text { height }\end{array}$ & Last value and date \\
\hline Processes-of-care & Diabetic foot examination, eye examination by ophtalmologist, influenza vaccination & Done/not done and date \\
\hline $\begin{array}{l}\text { Global satisfaction with patient } \\
\text { managment }\end{array}$ & Satisfaction with patients' care and management, barriers to better care management & \\
\hline \multicolumn{3}{|l|}{ Physicians' characteristics } \\
\hline Personal information & Age, gender, year of diploma, year of start of private practice, board certification type & \\
\hline Practice information & Practice location, practice type, activity rate, participation in quality programmes/circles & \\
\hline
\end{tabular}

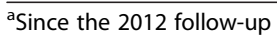


three contact attempts over different days and times is planned.

Because of time constraints and overall limited participation to research projects, treating physicians will be contacted every 2 years only.

\section{Follow-up questionnaires}

The core of the follow-up patients' questionnaires will be similar to the baseline questionnaire, particularly with regard to the questions targeting primary outcomes, healthcare utilisation, health status and health habits as well as to the questions assessing knowledge and participation to $\mathrm{PcD}$ projects. According to the needs and specific interests of the $\mathrm{PcD}$, new questions or thematic modules will be added to the follow-up questionnaires. In 2013 for example, a module on diabetic foot, as well as the Stanford self efficacy questionnaire and a health literacy question were added. In order to keep a questionnaire of a reasonable length, questions representing variables other than primary outcomes may be removed momentarily from the follow-up questionnaire.

The physicians' questionnaire will be similar to the baseline one.

\section{Data entry and analysis}

Upon receipt, questionnaires are first checked for obvious mistakes, and then scanned with an automated forms processing system, TeleForm ${ }^{\mathrm{Tm}}$. Exported data are then systematically verified to detect errors. Patients', physicians' and pharmacies' administrative data are kept in separate tables. A unique ID allows to link clinical and administrative information.

Descriptive analyses (univariate and bivariate) will be performed first. Then, depending on the research questions, appropriate statistical analyses will be conducted. Analyses, when appropriate, will take into account the hierarchical structure of the data (clustering by pharmacy and longitudinal design).

\section{Ethical considerations}

The study protocol was approved by the Cantonal Ethics Committee of Research on Human Beings of the Canton of Vaud (Protocol N $N^{\circ} 151 / 11$ ). This study is registered with ClinicalTrials.gov, identifier NCT01902043. Informed consent was obtained from all participants, and data will be kept anonymous.

\section{Discussion}

The implementation of CoDiab-VD followed a qualitative study on the evaluation of patients' and professionals' needs regarding the management of diabetes in this canton [23]. Both these first qualitative results and CoDiab-VD baseline quantitative results were, and will be useful for the adaptation of the PcD to the needs of healthcare professionals caring for patients with diabetes and other stakeholders. In addition, CoDiab-VD will be a helpful tool i) for the pragmatic evaluation of the $\mathrm{PcD}$, and ii) for the future development and implementation of PcD projects.

One of our study design's strength is the recruitment through community pharmacies [11], which allowed us to obtain a sample probably more representative of the population of patients with diabetes than a recruitment through medical practices or hospitals. In fact, we hypothesized that this method would limit the selection of patients on the basis of the level of care received, this information not being available to pharmacists. However, this hypothesis could not be confirmed by a comparison of participants' and non-participants' characteristics because data on non-participants could not be collected. Elements in favour of an acceptable representativeness are the fact that our study participants did not differ significantly from participants with diabetes of the CoLaus study [6], another population-based study conducted in the same region, regarding a few common characteristics (age, gender, education, smoking status, BMI) (P. MarquesVidal, personal communication). The minor differences probably stemmed from the fact that the population of the latter cohort were limited to a narrower age range (35-75). Another positive aspect of the cohort CoDiab-VD is the fact that we collected at baseline, and plan to collect in the future, a broad range of quality indicators that include not only commonly considered processes-of-care but also a variety of patient-reported outcomes. In fact, the latter encompass different aspects of diabetes and diabetes care that are important to patients but nevertheless often neglected despite the fact that they represent important measures to take into account when wishing to capture the complexity of the quality of diabetes care [24-26].

The cohort CoDiab-VD is, however, subject to several limitations. First, the number of patients recruited was below the sample size calculated (inclusion of 519 participants instead of the planed 600). However, because of a greater number of clusters (pharmacies) than expected and a conservative sample size calculation, the precision around point estimates was nevertheless acceptable. This sample size is also large enough (power $>90 \%$ ) to detect a $0.5 \%$ point decrease in HbA1C, a PCS or MCS change of 5 points, a PACIC change of 0.3 , or an absolute change of process of care of $10 \%$. Second, one could criticize the choice of self-reported data for the majority of the outcomes, since they may be prone to recall bias - an inherent limitation of such data collection, or be over- or underestimated. Specific analyses, on a fraction of the cohort data, demonstrated a good agreement between patient- and physician-reported outcomes for simple processes-of-care, such as measurement of blood pressure, HbA1C, weight and lipid profile [27]. Physician-reported 
outcomes were asked for in a second phase. Since their collection was dependent on the physicians' willingness to participate, these variables were only available for a fraction of the sample, about $50 \%$ at both recruitments and first follow-up. We will nevertheless favour the collection of some physician-reported measures (i.e. blood pressure, $\mathrm{HbA} 1 \mathrm{C}$ value) in the future because they are more accurate. Third, between the 2011 baseline recruitment and their first follow-up in 2012, 58 patients refused to continue to participate in the cohort, what could be explained by the lack of information of the 2011 initial group of patients about the annual follow-ups to come. Globally however, when considering patients both recruited in 2011 and in 2012, this amounted to only a tenth of the total baseline sample size. In addition, the comparison of characteristics of participants to the baseline and of participants to the 2013 follow-up did not highlight major differences.

CoDiab-VD will allow drawing a broad picture of patients with diabetes and their care over time. It will also contribute to the evaluation of the PcD and to support decisions about which targeted actions to implement.

\section{Additional file}

Additional file 1: Detailed description of the patients'

questionnaire. A comprehensive description of the patients'

questionnaire regarding the different variables and validated instruments

included, as well as their response options or scoring. (DOCX $40 \mathrm{~kb}$ )

\begin{abstract}
Abbreviations
ADDQoL: Audit of Diabetes-Dependent Quality of Life; AUDIT-C: Alcohol use disorders and identification test-consumption; AVD: Association Vaudoise du Diabète; CCM: Chronic Care Model; CDM: Chronic disease management; CHF: Swiss franc; CKD: Chronic kidney disease; CoDiab-VD: Cohort of patients with diabetes in the canton of Vaud; HbA1C: Glycated haemoglobin; HRQoL: Health-related quality of life; MCS: Mental component score; OAD: Oral anti-diabetic drug; PACIC: Patient Assessment of Chronic Illness Care; PCD: Programme cantonal Diabète; PCS: Physical component score; SF12: 12-Item Short-Form Health Survey.
\end{abstract}

\section{Competing interests}

The authors declare that they have no competing interests.

\begin{abstract}
Authors' contributions
EZ managed the study's follow-ups and wrote the manuscript. JB helped in the design of the study, managed the study recruitment and contributed to drafting the manuscript. BB participated in the conception and design of the study and contributed to drafting the manuscript. IPB conceived, designed and supervised the study, participated in the conduct of the study and in the writing of the manuscript. All authors read and approved the final manuscript.
\end{abstract}

\footnotetext{
Acknowledgements

We wish to thank all pharmacies, patients with diabetes and physicians who participated in the study. We also thank the partners and members of the working groups for their collaboration. We thank Mrs. Lucienne Boujon for copy editing the manuscript.

This project was funded by the Department of Public Health of the canton of Vaud ("Programme cantonal Diabète"). Prof. I. Peytremann-Bridevaux was supported by a grant from the Swiss National Science Foundation [PROSPER $\mathrm{N}^{\circ} 32333 \mathrm{~B}-123817$ and $\mathrm{N}^{\circ} 32333 \mathrm{~B}-139789$ ] and is currently supported by the Swiss School of Public Health + [Assistant Professorship grant].
}

Received: 30 January 2014 Accepted: 5 August 2015

Published online: 14 August 2015

\section{References}

1. Epping-Jordan JE, Pruitt SD, Bengoa R, Wagner EH. Improving the quality of health care for chronic conditions. Quality Safety Health Care. 2004;13(4):299-305.

2. McKee M, Nolte E. Responding to the challenge of chronic diseases: ideas from Europe. Clin Med. 2004;4(4):336-42.

3. Singh D, Ham C. Improving care for people with long-term conditions. A review of UK and international frameworks. Birmingham, UK: Health Services Management Center \& NHS Institute for Innovation and Improvement; 2006.

4. Wagner EH, Austin BT, Davis C, Hindmarsh M, Schaefer J, Bonomi A. Improving chronic illness care: translating evidence into action. Health Aff. 2001;20(6):64-78.

5. Whiting DR, Guariguata L, Weil C, Shaw J. IDF diabetes atlas: global estimates of the prevalence of diabetes for 2011 and 2030. Diabetes Res Clin Pract. 2011;94(3):311-21.

6. Firmann M, Mayor V, Vidal PM, Bochud M, Pecoud A, Hayoz D, et al. The CoLaus study: a population-based study to investigate the epidemiology and genetic determinants of cardiovascular risk factors and metabolic syndrome. BMC Cardiovasc Disord. 2008;8:6.

7. Hagon-Traub I, Hirsiger P, Bovet P, Ruiz J, Peytremann-Bridevaux I, Noth C, et al. Programme cantonal Diabète, présentation du programme. Lausanne: Service de la Santé Publique du canton de Vaud; 2010.

8. Nolte E, Saba H. DISMEVAL Developing and validating disease management evaluation methods for European healthcare systems: Final report. Santa Monica, CA: RAND Corporation; 2012.

9. Conklin A, Nolte E. Disease management evaluation: A comprehensive review of current state of the art. Santa Monica, CA: RAND Corporation; 2011.

10. Davidoff F, Batalden P, Stevens D, Ogrinc G, Mooney SE, group Sd. Publication guidelines for quality improvement studies in health care: evolution of the SQUIRE project. BMJ. 2009;338:a3152.

11. Peytremann Bridevaux I, Bordet J, Santschi V, Collet TH, Eggli M, Burnand B. Community-based pharmacies: an opportunity to recruit patients? Int J Public Health. 2012;58(2):319-22.

12. The Diabetes Control and Complications Trial Research Group. The effect of intensive treatment of diabetes on the development and progression of long-term complications in insulin-dependent diabetes mellitus. N Engl J Med. 1993;329(14):977-86.

13. Matthews DR. The natural history of diabetes-related complications: the UKPDS experience. United Kingdom Prospective Diabetes Study. Diabetes Obes Metab. 1999;2(1 Suppl):S7-13.

14. Shubrook Jr JH. Risks and benefits of attaining $\mathrm{HbA}(1 \mathrm{c})$ goals: examining the evidence. J Am Osteopathic Assoc. 2010;110(7 Suppl 7):eS7-12.

15. Ware Jr J, Kosinski M, Keller SD. A 12-Item short-form health survey: construction of scales and preliminary tests of reliability and validity. Med Care. 1996;34(3):220-33.

16. Bradley C, Todd C, Gorton T, Symonds E, Martin A, Plowright R. The development of an individualized questionnaire measure of perceived impact of diabetes on quality of life: the ADDQoL. Quality Res Int J Quality Aspects Treatment, Care Rehabil. 1999;8(1-2):79-91.

17. Glasgow RE, Whitesides H, Nelson CC, King DK. Use of the Patient Assessment of Chronic Illness Care (PACIC) with diabetic patients: relationship to patient characteristics, receipt of care, and self-management. Diabetes Care. 2005;28(11):2655-61.

18. Iglesias K, Burnand B, Peytremann-Bridevaux I. PACIC Instrument: disentangling dimensions using published validation models. Int J Quality Health Care. 2014;26(3):250-60.

19. Morisky DE, Green LW, Levine DM. Concurrent and predictive validity of a selfreported measure of medication adherence. Med Care. 1986;24(1):67-74.

20. Bush K, Kivlahan DR, McDonell MB, Fihn SD, Bradley KA. The AUDIT alcohol consumption questions (AUDIT-C): an effective brief screening test for problem drinking. Ambulatory Care Quality Improvement Project (ACQUIP). alcohol use disorders identification test. Arch Intern Med. 1998;158(16):1789-95.

21. Swiss Health Survey [http://www.bfs.admin.ch/bfs/portal/fr/index/infothek/ erhebungen_quellen/blank/blank/ess/04.html]

22. Whooley MA, Avins AL, Miranda J, Browner WS. Case-finding instruments for depression. Two questions are as good as many. J Gen Intern Med. 1997;12(7):439-45. 
23. Lauvergeon S, Burnand B, Peytremann-Bridevaux I. Chronic disease management: a qualitative study investigating the barriers, facilitators and incentives perceived by Swiss healthcare stakeholders. BMC Health Serv Res. 2012;12:176

24. Gibbons E, Fitzpatrick R. A structured review of patient-reported outcome measures for people with diabetes: an update. Oxford: National standards for diabetes self-management; 2009.

25. Calsbeek H, Ketelaar NA, Faber MJ, Wensing M, Braspenning J. Performance measurements in diabetes care: the complex task of selecting quality indicators. Int J Quality Health Care. 2013;25(6):704-9.

26. O'Connor PJ, Bodkin NL, Fradkin J, Glasgow RE, Greenfield S, Gregg E, et al. Diabetes performance measures: current status and future directions. Diabetes Care. 2011;34(7):1651-9.

27. Collet TH, Taffe P, Bordet J, Burnand B, Peytremann-Bridevaux I. Reproducibility of diabetes quality of care indicators as reported by patients and physicians. Eur J Pub Health. 2014;24(6):1004-9.

\section{Submit your next manuscript to BioMed Central and take full advantage of:}

- Convenient online submission

- Thorough peer review

- No space constraints or color figure charges

- Immediate publication on acceptance

- Inclusion in PubMed, CAS, Scopus and Google Scholar

- Research which is freely available for redistribution 